\title{
Integration of the Trigger and Data Acquisition systems in ATLAS
}

I. Riu ${ }^{1}$, M. Abolins ${ }^{2}$, P. Adragna ${ }^{3}$, G. Avolio ${ }^{4}$, S. Backlund ${ }^{4}$, E. Badescu ${ }^{5}$, S. Batreanu ${ }^{4,5}$, A. Battaglia ${ }^{6}$, H.P. Beck ${ }^{6}$, C. Bee ${ }^{7}$, P. Bell ${ }^{8}$, R.R. Blair ${ }^{9}$, A. Bogaerts ${ }^{2}$, T. Bold ${ }^{10}$, M. Bosman ${ }^{1}$, J. Boyd ${ }^{4}$, D. BurckhartChromek $^{4}$, M. Caprini ${ }^{5}$, D. Cimino ${ }^{11}$, M. Ciobotaru, ${ }^{4,5,10}$, A. Corso-Radu ${ }^{10}$, M.J. Costa ${ }^{12}$, R. Coura Torres ${ }^{4}$, R. Cranfield ${ }^{13}$, G. Crone ${ }^{14}$, J. Dawson ${ }^{9}$, J. De Almeida Simoes ${ }^{15}$, M. DellaPietra ${ }^{16}$, S. Demers ${ }^{17}$, A. Di Mattia ${ }^{2}$, M. Dobson ${ }^{4}$, A. Dos Anjos ${ }^{18}$, A. Dotti ${ }^{11}$, G. Drake ${ }^{9}$, N. Ellis ${ }^{4}$, Y. Ermoline ${ }^{2}$, I. Eschrich ${ }^{10}$, J. Ferland ${ }^{19}$, R. Ferrari ${ }^{20}$, M.L. Ferrer ${ }^{21}$, D. Francis ${ }^{4}$, S. Gadomski ${ }^{6}$, S. Gameiro ${ }^{15}$, H. Garitaonandia ${ }^{1}$, G. Gaudio ${ }^{20}$, B. Gorini ${ }^{4}$, S. Gowdy ${ }^{17}$, B. Green ${ }^{22}$, W. Haberichter ${ }^{9}$, H. Hadavand ${ }^{23}$, C. Haeberli ${ }^{6}$, M. Hauschild ${ }^{4}$, R. Hauser ${ }^{2}$, S. Hillier ${ }^{24}$, C. Hinkelbein ${ }^{25}$, R. Hughes-Jones ${ }^{8}$, J. Idarraga ${ }^{19}$, M. Joos ${ }^{4}$, A. Kazarov ${ }^{26}$, R. Kekoe ${ }^{23}$, G. Kieft ${ }^{27}$, J. Kirk ${ }^{22}$, S. Kolos ${ }^{10}$, K. Kordas ${ }^{6}$, K. Korcyl ${ }^{28}$, A. Kugel ${ }^{25}$, L. Leahu ${ }^{4,5}$, M. Leahu ${ }^{5}$, G. Lehmann Miotto ${ }^{4}$,

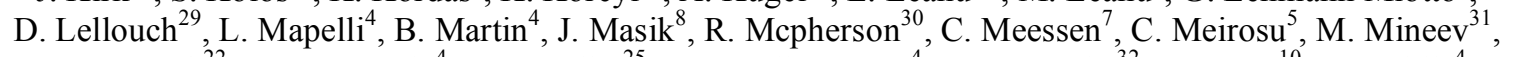
A. Misiejuk ${ }^{22}$, G. Mornacchi ${ }^{4}$, M. Mueller ${ }^{25}$, R. Murillo Garcia ${ }^{4}$, Y. Nagasaka ${ }^{32}$, A. Negri ${ }^{10}$, C. Padilla $^{4}$, F. Parodi ${ }^{33}$, E. Pasqualucci ${ }^{34}$, T. Pauly ${ }^{4}$, J. Petersen ${ }^{4}$, B. Pope ${ }^{2}$, P. Renkel ${ }^{23}$, C. Roda ${ }^{11}$, D. Salvatore ${ }^{35}$, D. Scannicchio ${ }^{20}$, C. Schiavii ${ }^{33}$, J. Schlereth ${ }^{9}$, I. Scholtes ${ }^{4}$, S. Sivoklokov ${ }^{36}$, J.E. Sloper ${ }^{4}$, I. Soloviev ${ }^{4}$, R. Spiwoks ${ }^{4}$, S. Stancu ${ }^{4,5,10}$, J. Stelzer ${ }^{4}$, J. Strong ${ }^{22 \dagger}$, S. Sushkov ${ }^{1}$, L. Tremblet ${ }^{4}$, G. Unel ${ }^{4,10}$, W. Vandellii ${ }^{4}$, J. Vermeulen ${ }^{27}$, J. Von Der Schmitt ${ }^{37}$, P. Werner ${ }^{4}$, S. Wheeler-Ellis ${ }^{10}$, F. Wickens ${ }^{14}$, W. Wiedenmann ${ }^{18}$,

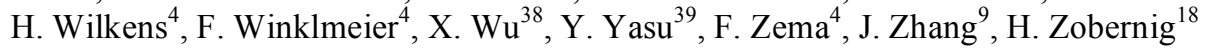

${ }^{1}$ Institut de Física d'Altes Energies (IFAE), Universitat Autònoma de Barcelona, Bellaterra, Spain

${ }^{2}$ Michigan State University, Department of Physics and Astronomy, East Lansing, Michigan, US

${ }^{3}$ Department of Physics, Queen Mary and Westfield College, University of London, London, UK ${ }^{4}$ CERN, Geneva, Switzerland

${ }^{5}$ National Institute for Physics and Nuclear Engineering, Institute of Atomic Physics, Bucharest, Romania

${ }^{6}$ Laboratory for High Energy Physics, University of Bern, Bern, Switzerland

${ }^{7}$ Centre de Physique des Particules de Marseille, IN2P3-CNRS, Marseille, France

${ }^{8}$ Department of Physics and Astronomy, University of Manchester, Manchester, UK

${ }^{9}$ Argonne National Laboratory, Argonne, Illinois, US

${ }^{10}$ University of California, Irvine, California, US

${ }^{11}$ Dipartimento di Fisica dell' Università di Pisa e I.N.F.N, Pisa, Italy

${ }^{12}$ Instituto de Física Corpuscular (IFIC), Universidad de Valencia, Valencia, Spain

${ }^{13}$ Department of Physics and Astronomy, University College London, London, UK

${ }^{14}$ Rutherford Appleton Laboratory, Chilton, Didcot, UK

${ }^{15}$ University Catolica-Figueira da Foz and University Nova de Lisboa, Lisbon, Portugal

${ }^{16}$ Dipartimento di Scienze Fisiche, Università di Napoli 'Federico II' e I.N.F.N., Napoli, Italy

${ }^{17}$ Stanford Linear Accelerator Center (SLAC), Stanford, US

${ }^{18}$ Department of Physics, University of Wisconsin, Madison, Wisconsin, US

${ }^{19}$ Department of Physics, McGill University, Montreal, Canada

${ }^{20}$ Dipartimento di Fisica Nucleare e Teorica dell' Università di Pavia e I.N.F.N., Pavia, Italy

${ }^{21}$ Laboratori Nazionali di Frascati dell' I.N.F.N., Frascati, Italy

${ }^{22}$ Department of Physics, Royal Holloway and Bedford New College, Egham, UK

${ }^{23}$ Department of Physics, Southern Methodist University, Dallas, Texas, US

${ }^{24}$ School of Physics and Astronomy, The University of Birmingham, Birmingham, UK

${ }^{25}$ Lehrstuhl fur Informatik V, Universitadt Mannheim, Mannheim, Germany

${ }^{26}$ Petersburg Nuclear Physics Institute (PNPI), St. Petersburg, Russia

${ }^{27}$ NIKHEF, Amsterdam, The Netherlands

${ }^{28}$ Henryk Niewodniczanski Institute of Nuclear Physics, Polish Academy of Sciences, Poland

${ }^{29}$ Department of Particle Physics, The Weizmann Institute of Science, Rehovot, Israel

${ }^{30}$ University of Victoria, Victoria, Canada

${ }^{31}$ Joint Institute for Nuclear Research, Dubna, Russia

${ }^{32}$ Hiroshima Institute of Technology, Hiroshima, Japan

${ }^{33}$ Dipartimento di Fisica dell' Università di Genova e I.N.F.N., Genova, Italy

${ }^{34}$ Dipartimento di Fisica dell' Università di Roma I 'La Sapienza'Roma e I.N.F.N., Roma, Italy

${ }^{35}$ Dipartimento di Fisica dell' Università della Calabria e I.N.F.N., Cosenza, Italy

${ }^{36}$ Moscow State University, Moscow, Russia

${ }^{37}$ Max-Planck-Institut fur Physik, Munchen, Germany

${ }^{38}$ Section de Physique, University of Geneva, Geneva, Switzerland

${ }^{39}$ KEK, High Energy Accelerator Research Organisation, Tsukub, Japan

${ }^{\dagger}$ deceased 
Abstract-During 2006 and spring 2007, integration and commissioning of trigger and data acquisition (TDAQ) equipment in the ATLAS experimental area has progressed. Much of the work has focused on a final prototype setup consisting of around eighty computers representing a subset of the full TDAQ system. There have been a series of technical runs using this setup. Various tests have been run including ones where around 6k Level-1 pre-selected simulated proton-proton events have been processed in a loop mode through the trigger and dataflow chains. The system included the readout buffers containing the events, event building, second level and third level trigger algorithms. Quantities critical for the final system, such as event processing times, have been studied using different trigger algorithms as well as different dataflow components.

\section{INTRODUCTION}

$\mathrm{T}$ he ATLAS experiment [1][2] is a general purpose protonproton detector designed to exploit the full discovery potential of the Large Hadron Collider (LHC) currently under construction at CERN. The goal of the ATLAS experiment is to explore the fundamental nature of matter and the basic forces that shape our universe. Its overall design is the result of the requirements of high precision muon momentum measurements, efficient tracking, large acceptance and very good electromagnetic calorimetry for electron and photon identification and measurements.

With a LHC bunch crossing rate of $40 \mathrm{MHz}$ and about 23 interactions per bunch crossing, a highly selective trigger system to reduce the expected $10^{9}$ interactions per second to an acceptable rate of a few hundred $\mathrm{Hz}$ is required. Sharing a large number of software components from the trigger event selection software to the offline physics analysis and reconstruction environment helps in understanding trigger efficiencies, and allows for a common development and run environment.

This paper describes the integration of the ATLAS Trigger and Data Acquisition (TDAQ) [3][4] systems. The architecture of the systems is shown in Fig. 1. As preparative work for the data taking phase, a full vertical slice of the final high level trigger and data acquisition chain, named preseries, has been installed in the ATLAS experimental zone. Trigger algorithms for both the second level (Level-2) and third level trigger (Event Filter) have been integrated and tested online in the pre-series setup. Level-1 pre-selected simulated data have been used and processed through the trigger and dataflow systems online in a loop mode.

\section{THE ATLAS TDAQ ARCHITECTURE}

\section{A. Trigger system description}

The ATLAS trigger is based on three levels of online selection: Level-1, Level-2, and Event Filter (EF). The second and third level triggers, together known as the High Level Trigger (HLT), are software based and implemented on
Personal Computers (PC) running the Linux operating system.

The Level-1 trigger [5][6] is implemented in custom hardware and reduces the initial event rate of $40 \mathrm{MHz}$ to about $75 \mathrm{kHz}$ as shown in Fig. 1. The Level-1 decision is based on data from the calorimeters and the muon detectors. For accepted events, small localized regions in pseudo rapidity and azimuthal angle centered on the high transverse momentum $\left(\mathrm{p}_{\mathrm{T}}\right)$ objects identified by the Level-1 trigger are determined. Each Region of Interest (RoI) associated with high $\mathrm{p}_{\mathrm{T}}$ candidate objects contains the type and the momentum threshold passed.

The Level-2 trigger selection process has to be capable of handling events at $75 \mathrm{kHz}$ up to $100 \mathrm{kHz}$ and with an average latency for the decision taking of the order of $10 \mathrm{~ms}$. It is guided by the RoI information supplied by the Level-1 trigger and gathered in custom made 9U VME boards, the RoI Builder (ROIB). It uses full granularity event data within a RoI from all detectors. In this way, only around $2 \%$ of the full event data are needed for the decision process at Level-2, thus reducing the required bandwidth to serve the Level-2 Trigger. The selection algorithms running in the Level-2 Processing Units (L2PU) request data from the ReadOut Buffers (ROB) for specific detectors in a Level-1 defined RoI for each processing step. The data are held in the ROBs until the Level-2 trigger accepts or rejects the event. The Level-2 event selection algorithms are controlled by the HLT selection framework and run inside the L2PU, each processing one event that has been assigned by the Level-2 SuperVisor (L2SV) application. The Level-2 output rate is about $3 \mathrm{kHz}$ with average event decision times of $10 \mathrm{~ms}$.

If an event is accepted by Level-2, the Event Builder (EB) collects all the event data fragments from the ROBs. The complete event is then made available to the EF for the final stage of trigger processing. Here, more complex algorithms provide a further rate reduction to about $200 \mathrm{~Hz}$ with typical event decision times of 1-2s. While the Level-2 reconstructs localized regions, the baseline for the EF is a full offline-like event reconstruction guided by the Level-2 result and

\section{ATLAS Trigger/DAQ system: architecture}

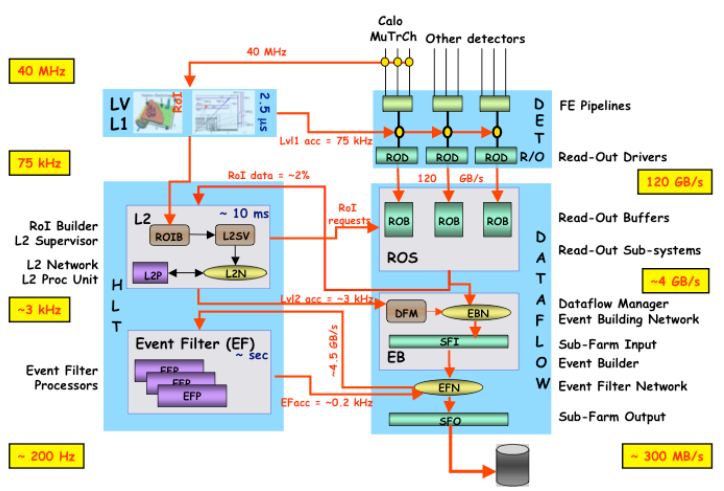

Figure 1 Drawing of the ATLAS Trigger and Data Acquisition systems architecture. 
operating at a rate of few $\mathrm{kHz}(\sim 3 \mathrm{kHz})$. It also uses more complete calibration, alignment and magnetic field data.

To achieve a fast rejection, the event processing in the HLT selection (both Level-2 and EF) proceeds in steps each including either feature extraction algorithms reconstructing useful quantities for triggering, or hypothesis algorithms rejecting or accepting according to conditions applied to these quantities. A sequence of steps in the HLT to trigger on a specific candidate like electron, photon, muon, etc. (named signature) is called slice. In order to ease the development of the trigger, ATLAS decided to re-use some elements of the offline framework inside the HLT. Among the advantages are the provision of the functions converting detector readout data to algorithms input by the detector experts. Additionally, it is easier to develop the HLT algorithms and perform trigger efficiency studies. Disadvantages include an increased dependence of HLT to offline software releases and the algorithms need to obey more severe constraints like timing and robustness. In spite of them, it has been found to be more advantageous than disadvantageous.

\section{B. DataFlow system description}

The DataFlow system [7] is responsible for moving the data that succeeded the Level-1 selection to the HLT, and transferring the accepted data to data storage. It is functionally decomposed in four building blocks: the ReadOut System (ROS), the RoI Collection, the EB and the Event Filter I/O.

The ROS is responsible for receiving data from the detector through its 1600 input links containing event fragments of an average size of $1 \mathrm{kB}$, forward them on request to Level-2 and $\mathrm{EB}$, and store the event data as long as it is explicitly told to delete them. The RoI Collection is responsible for gathering the data required by the Level-2 trigger while the EB is in charge of merging the event fragments coming from the ROS into a full event with an average size of 1.5 MB. The Event Filter I/O forwards events to the last selection stager, retrieves the accepted events from the EF and puts them on data storage.

\section{THE INTEGRATION TASK}

The ATLAS HLT algorithms are developed and tested in the offline framework. The integration task aims at making sure that these algorithms are working properly in the online environment giving identical results as if they were running in the offline framework. In order to ease this task, the integration work is decomposed in easily controllable steps to separate the problems that might appear from different environments.

Two command line tools emulating online running, one for Level-2 (called athenaMT [8]) and a similar one for EF (called athenaPT [9]) have been developed. These tools allow testing the HLT algorithms with the Level-2 and EF online applications without the need of running the ATLAS Data Acquisition system. Online and online emulation tools only differ in the raw data access. While running online, the data is sent through the network from one application to another, the online emulation tools emulate network access by delivering the data from memory.

The integration task is subdivided into different steps. As a first step, the online emulation tools are used to:

- modify or adapt the trigger offline configuration python script to be able to run online;

- make sure that offline and online emulation tools give exact trigger results event by event;

- make sure all trigger types including electrons, photons, muons, jets, taus, etc. work together in the online framework;

- make sure that all trigger algorithms are compatible with online operations, giving enough monitoring results, small output logfiles and meaningful online messages.

A second step includes running the trigger and Data Acquisition systems together, verifying the performance, measuring the algorithm processing time and comparing online to offline trigger results.

\section{PRE-SERIES LAYOUT AND TEST DESCRIPTION}

\section{A. Description of the pre-series layout}

The pre-series test bed setup [10] is a complete vertical slice of the ATLAS TDAQ system representing around $10 \%$ of the final system. The composition of this setup and the latest estimations for the final system are given in Table I. The preseries components were selected as rack mountable, $1 \mathrm{U}$ high end PCs. Each node has at least two gigabit network

TABLE I

NUMBER OF PCS IN THE PRE-SERIES AND FINAL SETUPS

\begin{tabular}{c|c|c}
\hline \hline Node & Pre-series setup & Final setup \\
\hline ROS & 12 & $\sim 150$ \\
L2SV & 2 & 12 \\
L2PU & 12 & $\sim 500$ \\
DFM & 2 & 12 \\
SFI & 6 & $\sim 100$ \\
EFP & 30 & $\sim 1900$ \\
SFO & 2 & $\sim 5$ \\
Online & 2 & 20 \\
Monitoring & 4 & 32 \\
File Servers & 5 & $\sim 80$ \\
\hline \hline
\end{tabular}


connections: one for the control and monitoring operations and another for data transfer to EB, Level-2 or EF networks. The ROS nodes, which need connection to both EB and Level-2 systems, were equipped with a single 4-port network interface card on PCI bus giving two times redundancy for data transfer. The ROS nodes, which receive up to 12 event fragments from different sections of the ATLAS detector are also equipped with the custom made PCI cards (ROBINs [11]) that will be used in the final system to receive and buffer these fragments. The event fragment data necessary for the studies can be preloaded into the ROBIN memory.

All computers apart from the ROS and ROIB VME crate are installed on the surface of the ATLAS experimental area. A total of around eighty PCs installed in six racks have been used in these studies. They are single core machines $3.2 \mathrm{GHz}$ Intel Xeon [12] or 2.4 GHz AMD Opteron [13].

\section{B. Description of the tests}

Different ATLAS Monte Carlo simulation data have been used in the tests. The Level-1 trigger has been simulated to the Monte Carlo events and only the succeeding events have been stored in raw data format for further processing. A total of around 6k Level-1 pre-selected events containing the Level-1 RoI information of a mixture of $\sim 55 \%$ jet-jet samples, $\sim 15 \%$ Wev, $13 \%$ W $\mu \nu, \sim 2 \%$ Zee, $\sim 3 \%$ Wtauhad, $~ 7 \% \mu \mu$ and $\sim 5 \%$ di-jets JF17 (dijets filtered to be very electromagnetic at generator level in order to get a large jet to electron fake rate) have been used. The sample contains information from all Level-1 RoI types, when applicable, allowing running any Level-2 and EF trigger slices. It is however not absolutely realistic since the Level-1 thresholds used are low. A second sample of around 4k Level-1 preselected ttbar events has also been used.

The Level-1 RoI information from the event is loaded into the ROIB and the detector information into the ROS system. Two different ways of starting the dataflow and trigger processes exist. The most commonly used one is by a Level-1 emulator application running in the ROIB VME crate that generates the trigger and starts processing events. A second possibility consists of using the L2SV application for loading the Level-1 information and triggering the events. On acceptance by Level-2, all data are passed through the EB to the EF farms. Finally the selected events are written to mass storage.

\section{Integrated trigger menu}

Different trigger algorithms have been integrated to be running online. They form what is named a trigger menu. They include algorithms for electron, photon, tau, jet and muon candidates selection. Different algorithms for each of the candidate types selection are implemented in both Level-2 and EF trigger slices. The trigger menu that has been integrated and summarized in Table II contains:

- the electron trigger slice starting with a Level-1 electromagnetic RoI of an energy greater than $7 \mathrm{GeV}$;

- the photon trigger slice starting with the same Level-1 RoI as the electron slice;

- $\quad$ the jet trigger slice starting with a Level-1 jet RoI with an energy greater than $15 \mathrm{GeV}$;

- the muon trigger slice starting with a Level-1 muon RoI with an energy greater than $6 \mathrm{GeV}$;

- and the tau trigger slice starting wit a Level-1 tau RoI with an energy greater than $8 \mathrm{GeV}$.

\section{RESUlts}

\section{A. Online and offline trigger algorithm results comparison}

Online tests processing the mixed events sample through the Level-2 and EF trigger slices have taken place since mid 2006. The trigger algorithm results have been compared between online and offline both in a statistical manner and event by event.

Examples of results from the trigger algorithms that have been compared online and offline are shown in Fig. 2, 3 and 4. Fig. 2 and 3 show distributions taken online of the electromagnetic clusters energy and track transverse momentum reconstructed per RoI by two different Level-2 algorithms respectively. Fig. 4 shows the number of events succeeding a Level-2 trigger step when running offline. It shows how the events are rejected as they are being processed through the five steps in the Level-2 tau trigger slice. This slice contains three steps being hypothesis algorithms rejecting events while the other two are used to extract useful features for cutting. The plot shows that the last hypothesis is not cutting any event in this particular sample since they were all already cut in the second hypothesis.

TABLE II

INTEGRATED TRIGGER MENU INCLUDING THE THRESHOLDS (THR.)

\begin{tabular}{|c|c|c|c|c|}
\hline & Egamma & Jet & Muon & Tau \\
\hline $\begin{array}{c}\text { Level-1 } \\
\text { thr. }\end{array}$ & $\begin{array}{c}\text { EM01 } \\
7 \mathrm{GeV} \text { thr. }\end{array}$ & $\begin{array}{c}\text { JT15 } \\
15 \mathrm{GeV} \text { thr. }\end{array}$ & $\begin{array}{c}\text { MU06 } \\
6 \mathrm{GeV} \text { thr. }\end{array}$ & $\begin{array}{c}\text { HA08 } \\
8 \mathrm{GeV} \text { thr. }\end{array}$ \\
\hline Level-2 & $\begin{array}{c}\text { e10 } \\
\text { signature } \\
\text { g10 } \\
\text { signature }\end{array}$ & $\begin{array}{c}\text { jet20 } \\
\text { signature }\end{array}$ & $\begin{array}{c}\text { mu6 } \\
\text { signature }\end{array}$ & $\begin{array}{c}\text { tau10 } \\
\text { signature }\end{array}$ \\
\hline $\mathrm{EF}$ & $\begin{array}{c}\text { e10 } \\
\text { signature } \\
\text { g10 } \\
\text { signature }\end{array}$ & $\begin{array}{c}\text { jet20 } \\
\text { signature }\end{array}$ & $\begin{array}{c}\text { mu6 } \\
\text { signature }\end{array}$ & $\begin{array}{c}\text { tau } 10 \\
\text { signature }\end{array}$ \\
\hline
\end{tabular}




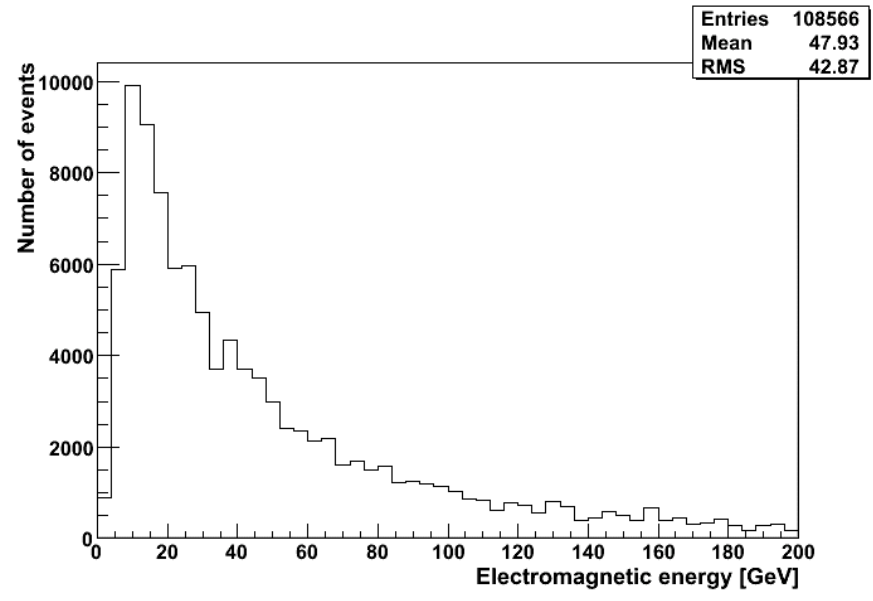

Figure 2 Online distribution of the electromagnetic energy of the clusters reconstructed per RoI in the mixed events sample by the Level-2 clustering algorithm.

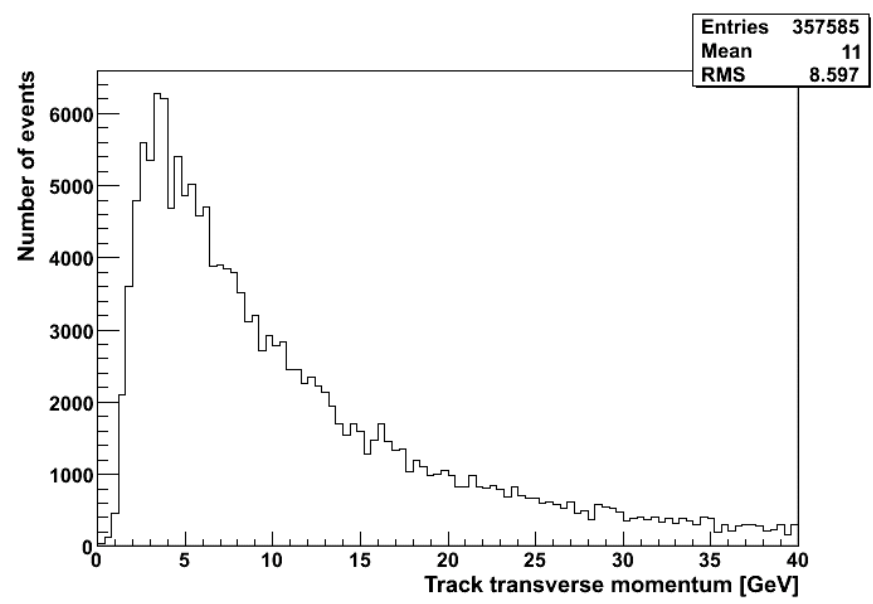

Figure 3 Online distribution of the transverse momentum of the tracks reconstructed per RoI in the mixed events sample by the Level-2 tracking algorithm.

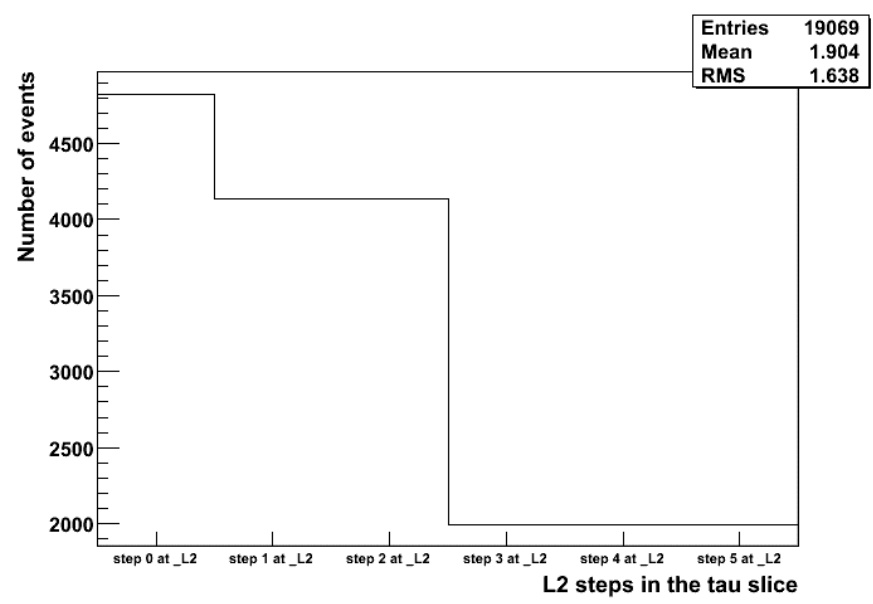

Figure 4 Offline distribution showing the number of events succeeding each Level-2 step when running the tau slice over the mixed events sample. Each bin corresponds to one step.
These distributions together with some others have been compared running online, running offline and running the online emulation tools. They have been found to be identical. At the same time, trigger algorithm results have been compared event by event in both Level-2 and EF giving identical results as well.

\section{B. Level-2 trigger algorithm time results}

As important as knowing that trigger studies results obtained offline are also obtained online, is checking the time constraints of both Level-2 and EF trigger selections. Running the egamma (electron and photon combined) trigger slice (described in [14]), the time it takes for the Level-2 trigger algorithm to compute, for each RoI, the energy of the electromagnetic clusters after getting all necessary information from the mixed events sample (T2CaloEgamma algorithm) is shown in Fig. 5 and has a mean value of $7.4 \mathrm{~ms}$. Running the muon trigger slice (described in [15]), the mean time it takes to reconstruct a track in the muon spectrometer per RoI (muFast algorithm) is found to be $6.2 \mathrm{~ms}$, as shown in Fig. 6 .

Taking into account that the average number of RoIs per event is expected to be of around 1.5, both algorithms are within a $\sim 10 \mathrm{~ms}$ time interval. However, time spent in other feature extraction and hypothesis algorithms included in these slices needs to be taken into account. The $10 \mathrm{~ms}$ Level-2 constraint was estimated in 2003 for expected single core computers of $8 \mathrm{GHz}$ clock frequency, which do not exist. The computers that are going to be used will be $4.2 \mathrm{GHz}$ processors with four cores, where each of them can take $\sim 40 \mathrm{~ms}$ to achieve the same designed throughput. We have, therefore, confidence that the new constraint will be fulfilled specially taking into account that the mixed events simulated data sample used is not completely representative of what Level-1 trigger will be giving in reality.

\section{EF trigger algorithm time results}

The EF trigger algorithms are executed online for accepted Level-2 events and the time to run a complete slice is recorded. Fig. 7 and 8 show the online time distributions to run the complete jet and tau slices respectively through the accepted Level-2 mixed events sample. The different peaks in Fig. 7 correspond to events having different number of Level-2 RoIs. The EF jet and tau slices are run with a mean time of $122.9 \mathrm{~ms}$ and $226.5 \mathrm{~ms}$ respectively, fulfilling the requested $1 \mathrm{~s}$ allowed time for EF algorithms.

\section{ATLAS event display}

In parallel to data taking, the ATLAS event display has been made to work online. One of the EF computers was running the ATLAS offline reconstruction and event display. A real cosmic muon event is shown in Fig. 9. 


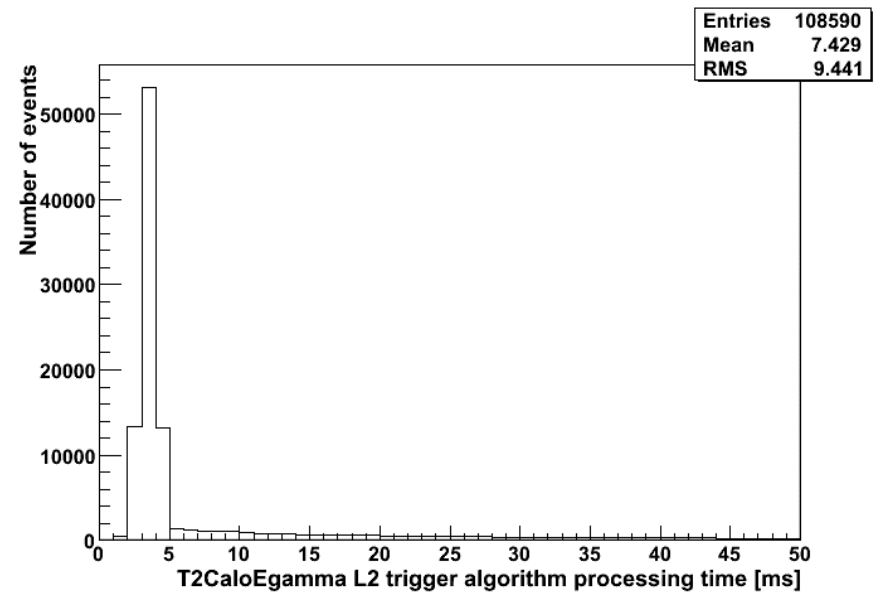

Figure 5 Online distribution of the time it takes to run the Level-2 electromagnetic cluster reconstruction trigger algorithm (T2CaloEgamma) through the mixed events sample.

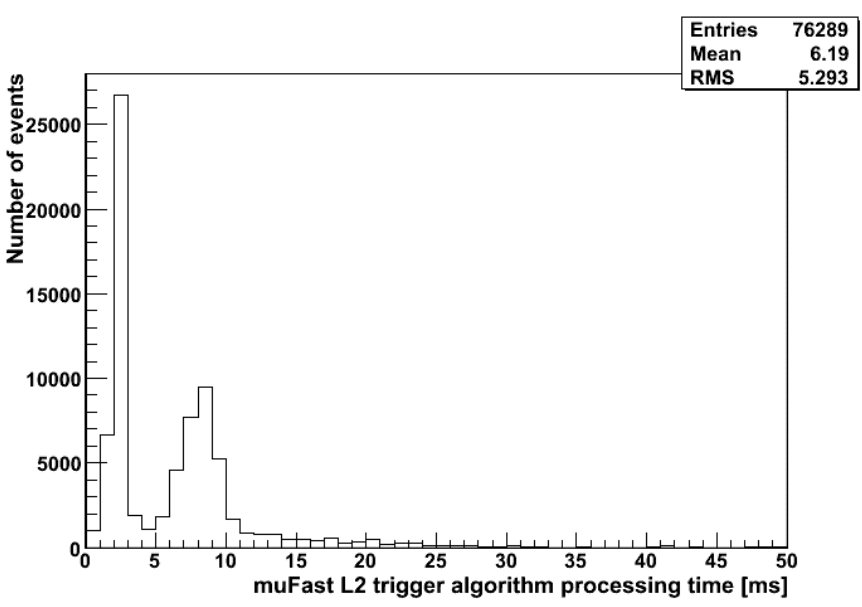

Figure 6 Online distribution of the time it takes to run the Level-2 muon track reconstruction trigger algorithm (muFast) through the mixed events sample.

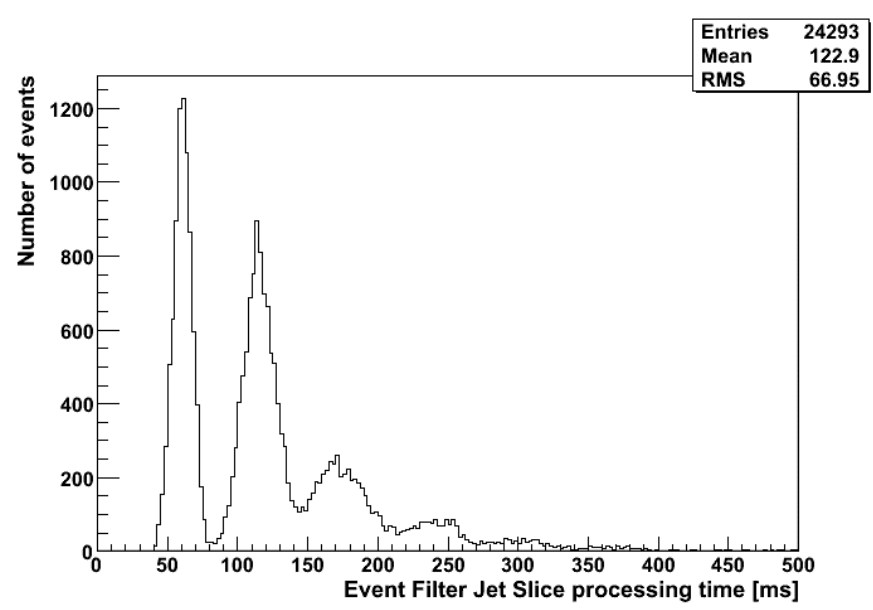

Figure 7 Online distribution of the time it takes for the EF jet slice to run through the selected Level-2 mixed events sample.

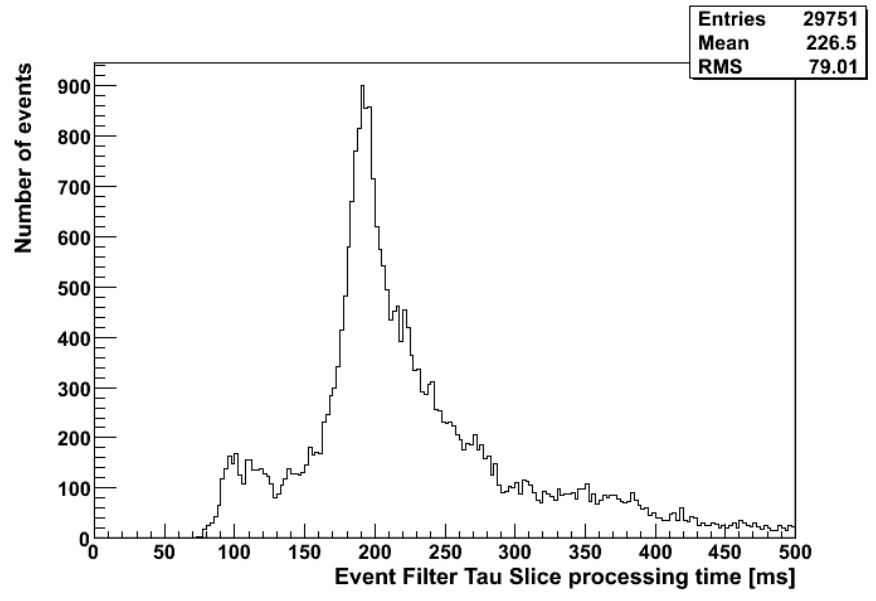

Figure 8 Online distribution of the time it takes for the EF tau slice to run through the selected Level-2 mixed events sample.

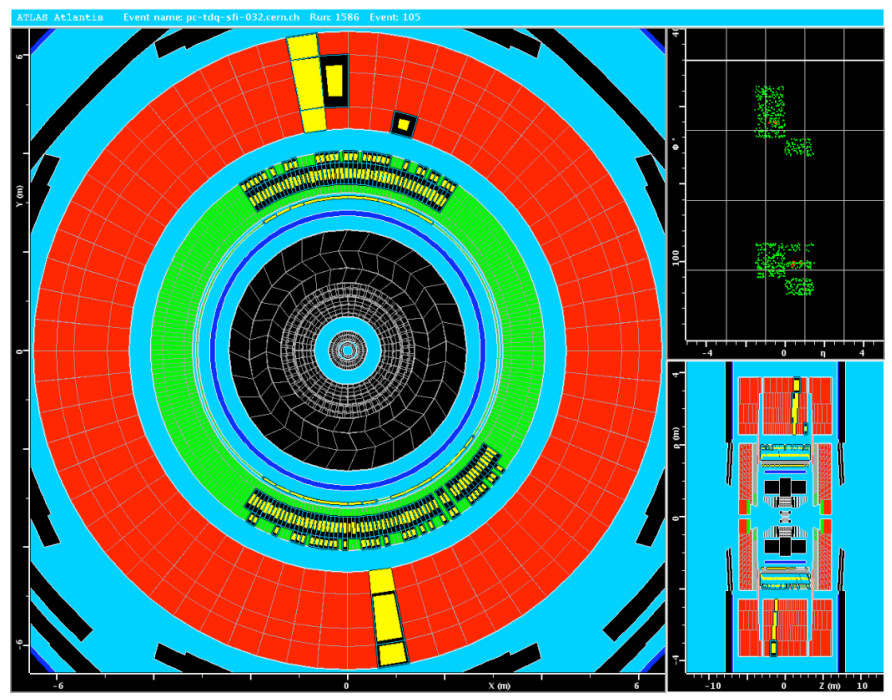

Figure 9 ATLAS cosmic muon event display taken online.

\section{CONCLUSIONS}

The ATLAS Trigger and Data acquisition systems have been successfully integrated. Integration tests involving a system of around eighty computers have taken place several times since mid 2006. Identical trigger algorithm results have been obtained running online and offline. HLT algorithm execution times are expected to be within the allocated time budget. Finally, the ATLAS commissioning with cosmic events in the near future will profit from the integration work. More computers for the high level trigger and data acquisition systems will be acquired during 2007 in preparation for the data taking phase and similar tests will be performed.

\section{ACKNOWLEDGMENT}

The authors would like to thank the ATLAS Trigger Algorithms, Performance and Menu group for providing the trigger algorithms and slices to be integrated online. 


\section{REFERENCES}

[1] ATLAS Collaboration, Technical Proposal for a General Purpose pp Experiment at the Large Hadron Collider at CERN, CERN/LHCC/9343, LHCC/P2, 15 December 1994.

[2] ATLAS Collaboration, ATLAS Detector and physics performance Technical Design Report, CERN/LHCC/99-14, 25 May 1999.

[3] ATLAS Collaboration, ATLAS High-Level Trigger, Data Acquisition and Controls, Technical Design Report, ATLAS TDR-016, CERN/LHCC/2003-022, 30 June 2003.

[4] B. Gorini et al., The ATLAS Data acquisition and High-Level Trigger: concept, design and status, proceedings of CHEP 2006, 13-17 Feb 2006, Mumbai, India.

[5] ATLAS Collaboration, ATLAS Level-1 Trigger, Technical Design Report, ATLAS TDR-012, CERN/LHCC/1998-014, 24 June 1998.

[6] D. Berge et al., Status of the ATLAS Level-1 Central Trigger and Muon Barrel Trigger and First Results from Cosmic-Ray Data, proceedings of IEEE Real Time 2007 and submitted to IEEE Trans. Nucl. Sci.

[7] H.P. Beck et al, Performance of the final Event Builder for the ATLAS Experiment, proceedings of IEEE Real Time 2007 and submitted to IEEE Trans. Nuc. Sci.

[8] S. Armstrong et al., Studies for a common selection software environment in ATLAS: from the level-2 trigger to the offline reconstruction, IEEE Trans. Nuc. Sci., 51 (2004) 915-920.

[9] S. Sushkov et al., Development and Tests of the Event Filter for the ATLAS Experiment, proceedings of IEEE Real Time 2005, 4-10 June 2005, Stockholm, Sweden.

[10] N. G. Unel et al., Studies with the ATLAS Trigger and Data Acquisition 'pre-series' setup, proceedings of CHEP 2006, 13-17 Feb 2006, Mumbai, India.

[11] B. Green et al., ATLAS trigger/DAQ RobIn prototype, IEEE Trans. Nuc. Sci. 51 (2004) 465-469.

[12] Intel Corporation, Xeon processor, http://www.intel.com/xeon .

[13] AMD Corporation, Opteron processor, http://www.amd.com/opteron .

[14] C. Schiavi et al., Implementation and performance of the high level trigger electron and photon selection for the ATLAS Experiment at the LHC, proceedings of the IEEE NSS 2004, 16-22 October 2004, Rome, Italy.

[15] A. Sidoti et al., The ATLAS muon trigger slice, proceedings of IEEE Real Time 2007 and submitted to IEEE Trans. Nucl. Sci. 